\title{
Aspects morphologiques des erreurs chromosomiques létales
}

\author{
J Boué1, R Bessis² \\ 1 INSERM U73, Château de Longchamp, 75016 Paris; \\ ${ }^{2}$ Centre d'Échographie de l'Odéon, 122, bd Saint-Germain, 75006 Paris, France
}

(28 Réunion de la Société Française pour l'Étude de la Fertilité; Paris, 19-21 octobre 1989)

\begin{abstract}
Résumé - Les examens chromosomiques effectués sur les tissus d'avortements spontanés ont permis d'établir des corrélations entre la constitution chromosomique et l'aspect des produits d'expulsion, c'est-à-dire entre caryotype et phénotype. Les critères utilisés pour déterminer le phénotype sont basés sur l'estimation du stade de développement atteint par l'œuf, l'aspect macroscopique de l'embryon et du placenta en tenant compte des modifications morphologiques liées à la rétention in utero. La probabilité qu'une anomalie chromosomique soit la cause de l'avortement est élevée quand la mort de l'œuf se situe à un stade précoce, c'est-à-dire avant 5-6 semaines de développement et que l'expulsion est différée. La fréquence des anomalies chromosomiques est estimée à $75 \%$ quand l'œuf est arrêté à 2-3 semaines de développement et $65 \%$ quand l'œuf atteint 5 à 6 semaines. Nous avons tenté de voir s'il était possible à partir des signes anatomiques décrits de soupçonner dès l'échographie l'anomalie chromosomique en cause.
\end{abstract}

anomalies chromosomiques fcetales / phénotype / ultrasons

Summary - Morphological aspects of lethal chromosomal abnormalities. Correlations have been established between phenotype and karyotype in spontaneously aborted specimens. The criteria used to determine the phenotype were based on estimated developmental age and macroscopic examination of embryo and placenta, taking into account the morphological modifications due to in utero retention. The probability that a chromosomal aberration was the cause of the abortion is high when the developmental arrest occurred before 5-6 weeks and the expulsion was delayed. The frequency could be estimated at $75 \%$ when the developmental arrest was at 2-3 weeks, and $65 \%$ when the developmental stage reached 5-6 weeks. Main anatomical features are described permitting an attempt to assign the type of chromosomal anomaly by ultrasonographic examination.

\section{fetal chromosomal anomaly / phenotype / ultrasonography}

Les examens chromosomiques effectués sur les tissus d'avortements spontanés ont permis d'établir des corrélations entre la constitution chromosomique et l'aspect des produits d'expulsion, c'est-à-dire entre caryotype et phénotype (Boué et al, 1976).

Les critères utilisés pour déterminer le phénotype sont basés sur l'estimation du stade du développement atteint par l'œuf, l'aspect macroscopique de l'embryon et du placenta en tenant compte des modifications morphologiques liées à la rétention in utero.

Cette période de rétention est relativement constante, de 5 semaines en moyenne, et correspond à la durée de maintien de la grossesse par le système hormonal maternel. La probabilité qu'une anomalie chromosomique soit la cause de 
l'avortement est élevée quand on assiste à la mort de l'œuf à un stade précoce, c'està-dire avant 5 à 6 semaines de développement, et que l'expulsion est différée. La fréquence de telles anomalies est estimée à $75 \%$ quand l'œuf est arrêté à 2-3 semaines de développement et à $65 \%$ quand l'œuf atteint 5- 6 semaines.

Nous avons tenté de voir s'il était possible, en partant des données anatomiques déjà établies pour les différents caryotypes, de soupçonner dès l'échographie l'anomalie chromosomique en cause. D'emblée, certaines difficultés doivent être mentionnées, liées aux faits que l'échographie intervient à un moment aléatoire, et qu'il est rarement possible de dater l'arrêt de l'évolution ou d'évaluer la durée de la rétention.

Les informations cliniques, même scrupuleusement recueillies, ne sont pas déterminantes. La pratique montre bien que l'arrêt de l'évolution est longtemps asymptomatique et qu'il est fréquemment une découverte échographique. Les dosages hormonaux plasmatiques ne peuvent être utilisés ni pour dater une grossesse ni pour en estimer le pronostic. La longueur cranio-caudale embryonnaire constatée ne peut être utilisée pour déterminer le moment de l'arrêt évolutif, car celui-ci a pu être précédé d'une phase de ralentissement de croissance plus ou moins longue, plus ou moins marquée.

\section{ÉLÉMENTS ÉCHOGRAPHIQUES}

L'utilisation de capteurs endo-vaginaux permet une approche plus performante des œufs intra-utérins dans les premières semaines. Le sac gestationnel peut souvent être repéré deux semaines après la fécondation. Une semaine plus tard, l'embryon est visualisé et avant la fin du premier mois de gestation, l'activité cardiaque est enregistrable.

La vésicule vitelline est très rapidement identifiée, et c'est à sa surface que l'embryon pourra être reconnu comme une petite formation linéaire, fortement échogène. La cavité amniotique, qui contient l'embryon, est sphérique et son contour est parfaitement individualisable de la poche choriale.

Au décours de l'arrêt de son développement, l'œuf peut demeurer "en l'état" ou subir certaines dégradations morphologiques. L'embryon devient moins échogène, ses limites moins précises. Il est alors plus difficilement repérable et pourrait échapper à un examen sommaire. Le sac amniotique se réduit et il est plus délicat de la distinguer de l'espace extra-amniotique. Plus tardivement, le trophoblaste perd son homogénéité. Ses contours prennent un aspect irrégulier, tourmenté, et des plages plus ou moins anéchogènes apparaissent.

Fig 1. Tétraploïdie : a) petit œứ de $8 \mathrm{~mm}$ enchassé dans la caduque; b) œuf constitué de la vésicule choriale seule; sans amnios; c) aspect échographique à 8 semaines d'aménorrhée.

Fig 2. CEuf clair : a) œuf de $35 \mathrm{~mm}$, cavité amniotique fermée de $20 \mathrm{~mm}$ sans formation embryonnaire - trisomie 2; b) aspect échographique de l'œuf clair; photo et schéma (u: utérus; vc: vésicule choriale; ca: cavité amniotique).

Fig 3. CEuf intramuqueux : a) œuf de $14 \mathrm{j}$ (non caryotypé) trophoblaste hypoplasique, pas d'ébauche embryonnaire, biopsie d'endomètre (E Philippe); b) aspect échographique : photo et schéma (u: utérus; m: muqueuse utérine; cu: cavité utérine; o: œuf).

Fig 4. Trisomie $16:$ a) œuf en quenouille de $40 \mathrm{~mm}$ de grand axe, vésicule choriale vaste avec formation embryonnaire minime (disque embryonnaire), petite cavité amniotique ou vésicule vitelline hématome intrachorial diffus; b) aspect échographique : photo et schéma (u: utérus; t: trophoblaste; e: embryon; cu: cavité utérine). 

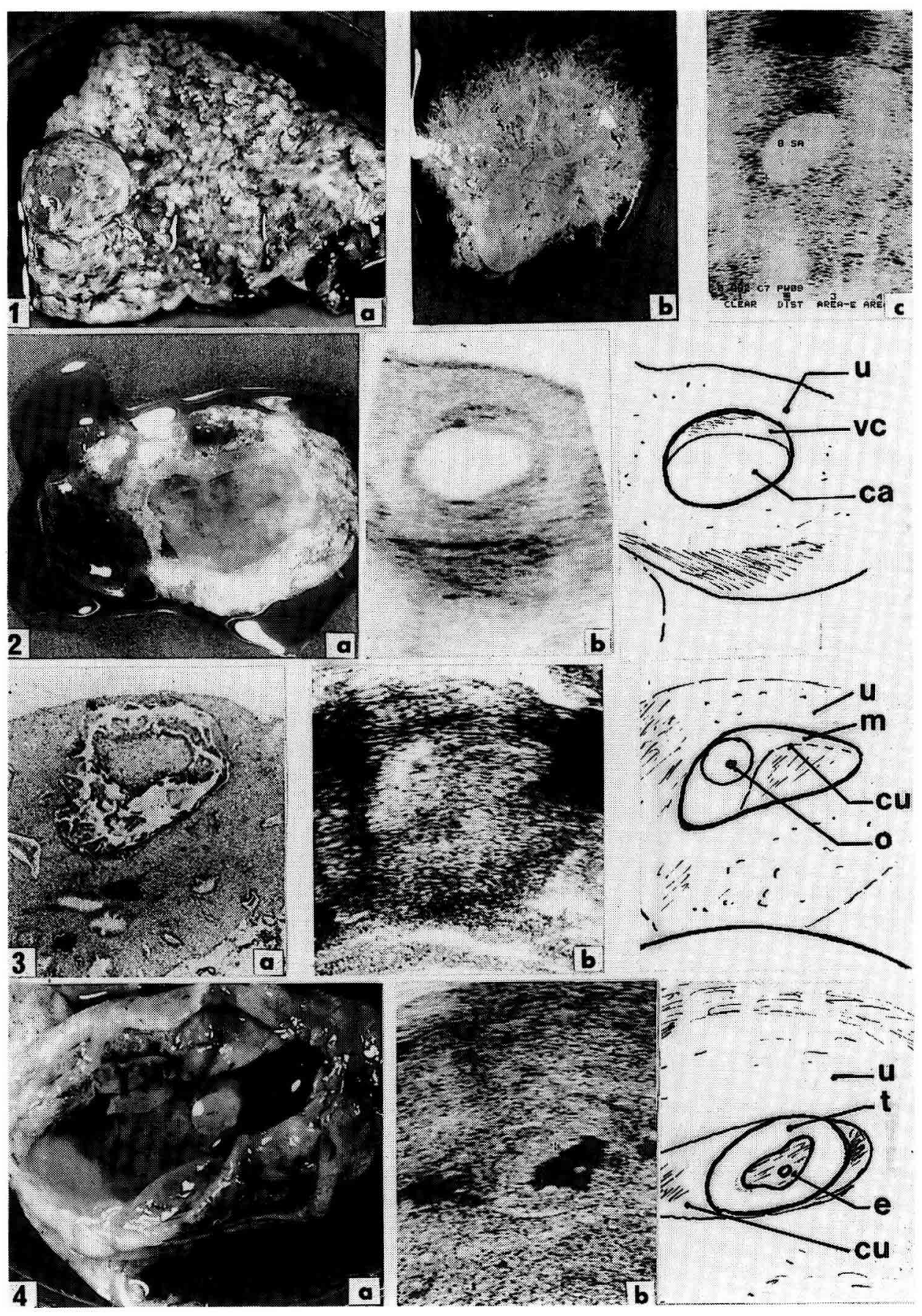


\section{ASPECTS MORPHOLOGIQUES}

\section{Arrêt d'évolution à 2-3 semaines de développement (4-5 semaines d'aménorrhée)}

1) Si l'œuf est constitué d'une vésicule choriale seule (fig 1), sans revêtement amniotique, le diagnostic le plus probable est celui d'une tétraploïdie : l'œuf a 92 chromosomes. Il y a eu division chromosomique sans division cytoplasmique. La multiplication cellulaire est possible pendant un certain temps mais l'œuf s'arrête d'évoluer à un stade très précoce. Ce caryotype correspond à $5 \%$ des anomalies chromosomiques; 2) l'œuf est constitué d'une vésicule choriale avec cavité amniotique, sans formation embryonnaire : c'est l'œuf clair classique (fig 2 ). La fréquence des anomalies chromosomiques est de $70 \%$ environ. II s'agit principalement de trisomies des chromosomes grands et moyens; chromosomes $2,4,5$ et 6 à 12 . L'œuf clair représente la moitié des phénotypes des anomalies chromosomiques; 3) les monosomies autosomiques, dont le nombre est théoriquement équivalent à celui des trisomies conçues, ne sont jamais trouvées dans les produits d'avortement. II est maintenant démontré qu'elles sont expulsées avant l'implantation.
II est probable que quelques œufs monosomiques arrivent à s'implanter. Mais l'arrêt de leur développement est sans doute immédiat. Le volume ovulaire est alors si modeste que le repérage échographique n'en est pas réellement envisageable. II arrive parfois que l'on puisse identifier une petite structure, évocatrice mais sans caractères spécifiques, enchassée dans la caduque (fig 3). Le contexte, les éléments évolutifs, cliniques et hormonaux, permettent de la rattacher à une grossesse débutante. En pratique, le diagnostic est plus souvent posé à l'occasion de biopsies d'endomètre.

\section{Arrêt de l'évolution à 3-4 semaines de développement (5-6 semaines d'aménorrhée)}

On observe une vaste vésicule choriale, une petite formation embryonnaire, sous forme de disque embryonnaire, et une petite cavité amniotique ou vésicule vitelline (fig 4). C'est le phenotype de la trisomie 16 qui est la plus fréquente des trisomies à l'origine des avortements puisqu'elle correspond à un tiers des trisomies autosomiques.

Fig 5. a) trisomie 13 : spécimen arrêté à 5 semaines de développement, petit embryon malformé, extrémité céphalique réduite, vaste cavité amniotique de $5 \mathrm{~cm}$; b) trisomie $14:$ spécimen de 6 semaines de développement, embryon de $42 \mathrm{j}$, ouverture de la face, placenta hypoplasique; c) trisomie 21 : cavité amniotique de $25 \mathrm{~mm}$, embryon de $40 \mathrm{j}$, extrémité céphalique peu développée; d) trisomie 9 : embryon de 38-40 j, hématome intrachorial diffus.

Fig 6. Monosomie $X$ : a) gémellaire $45, X O$, œufs reçus fermés; à l'ouverture, cordons avec débris embryonnaires macérés à leur extrémité, sans embryons (2/3 des observations); b) œuf de $60 \mathrm{~mm}$ avec embryon sans malformations apparentes, de 6 semaines de développement (1/3 des observations); c) aspects échographiques : photo et schéma (t: trophoblaste; e: embryon; ca: cavité amniotique; vv: vésicule vitelline).

Fig 7. Triploïdie : a) œuf de $50 \mathrm{~mm}$, placenta avec vésicules pseudo-molaires de taille variable et hématome intrachorial, petit embryon dont l'extrémité céphalique est conique (triploïdie par dispermie); b) aspect du placenta avec dégénérescence molaire plus importante (triploïdie par dispermie); c) aspect des triploïdies par digynie, embryon de 6 semaines, placenta hypoplasique; d) dessin de Cruveilhier (1839) : môle embryonnée évoquant une triploïdie. 

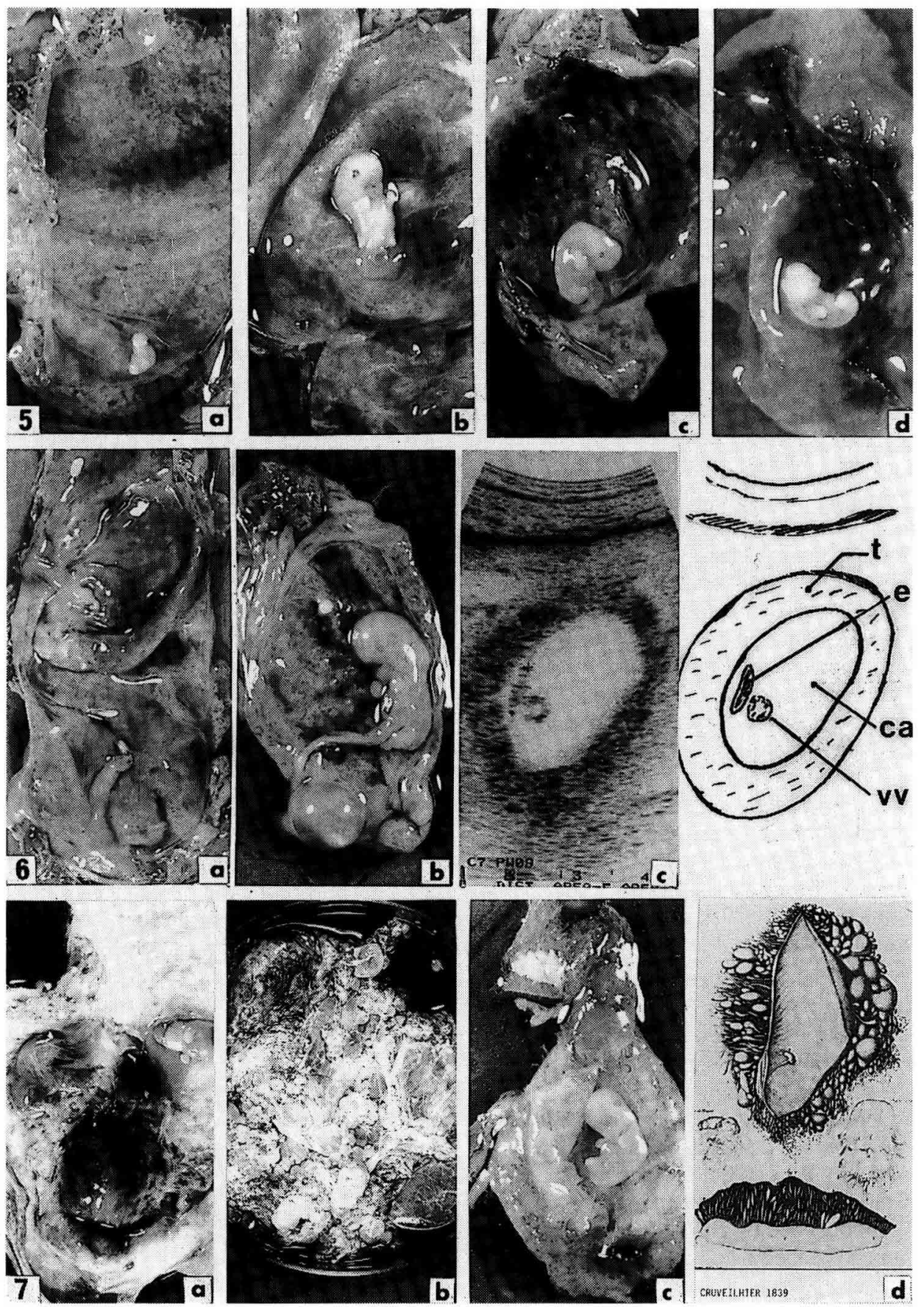


\section{Arrêt de l'évolution à 5-6 semaines de développement (7-8 semaines d'aménorrhée)}

II existe une formation embryonnaire minime, mesurant 2 à $10 \mathrm{~mm}$. lci sont rassemblées les trisomies $D, 13,14$ et 15 , la plupart des trisomies 18 , les trisomies 21 et 22 , et les monosomies $X$. Dans les trisomies, le volume amniotique est variable, souvent important par rapport à l'embryon, car la cavité amniotique continue à se développer après la mort de l'embryon (fig 5). Le chorion est hypoplasique, des hématomes intrachoriaux, sous-amniotiques, se sont constitués au cours de la rétention. L'embryon est souvent malformé, généralement au niveau de l'extrémité céphalique. Les monosomies $X$ (fig 6 ) sont très fréquentes dans les produits d'avortement $(20 \%$ des anomalies chromosomiques). Le phénotype est caractéristique dans les 2/3 des cas : il s'agit d'un œuf de 6 semaines de développement, fermé. A l'ouverture de la cavité amniotique, il existe un cordon à l'extrémité duquel on constate des débris embryonnaires macérés minimes, l'embryon est absent, probablement résorbé. Dans 1/3 des observations, l'embryon est présent sans malformation évidente, il a un développement également de 6 semaines. Les hématomes intrachoriaux sont constants dans tous les cas.

Les môles embryonnées (fig 7) : l'embryon est petit ( 5 à $6 \mathrm{~mm}$ ) malformé, son extrémité céphalique apparaît conique. La cavité amniotique est vaste et le placenta présente en certaines zones une dégénérescence pseudo-molaire. Les vésicules molaires sont de taille très variable, donnant un aspect hétérogène au placenta. Cet aspect correspond aux triploïdies, l'œuf possède 69 chromosomes. II existe 3 jeux chromosomiques dont 2 sont d'origine paternelle et 1 d'origine maternelle. Cette anomalie s'est constituée par la fécondation de l'ovule par 2 spermatozoïdes. Trois quarts des triploïdies relèvent de ce mécanisme. Dans les cas de triploïdies par digynie ( 2 jeux chromosomiques maternels et 1 jeu chromosomique paternel), l'aspect de l'cuuf est différent. II n'y a pas de dégénérescence pseudo-molaire mais une hypoplasie placentaire. On ne retrouve pas de caractéristique échographique permettant de distinguer le spécimen triploïde de certaines trisomies ou monosomies $\mathrm{X}$.

La môle hydatiforme n'est pas fréquente dans nos régions. C'est la seule anomalie chromosomique qui ait une distribution géographique îles du Pacifique, Sud-Est asiatique, Sénégal). Le placenta est constitué de vésicules molaires de taille variable et il n'y a jamais de cavité amniotique ni de formation embryonnaire. La constitution chromosomique est habituellement $46, \mathrm{XX}$ et correspond à 2 jeux chromosomiques paternels identiques, sans jeu chromosomique maternel. Ceci résulte de la fécondation d'un ovocyte, dont le noyau a été expulsé avec le second globule polaire, par un spermatozoïde dont le noyau se dédouble ensuite pour reconstituer un spécimen diploïde à 46 chromosomes.

Les môles hydatiformes $46, Y Y$ ne sont pas décrites, car cette constitution chromosomique est certainement très rapidement létale. Quelques môles sont $46, X Y$. Les 2 jeux chromosomiques sont toujours d'origine paternelle (fécondation par 2 spermatozoïdes ou par 1 spermatozoïde diploïde).

\section{RÉFÉRENCE}

Boué J, Philippe E, Girouḍ A, Boué A (1976) Phenotypic expression of lethal chromosomal anomalies in human abortuses. Teratology 14, 3-19 